\title{
Cytokines and integrins related to inflammation of joint and gut in patients with spondyloarthritis and inflammatory bowel disease
}

\author{
Ewa Kontny ${ }^{1}$, Joanna Dmowska-Chalaba², Brygida Kwiatkowska², Włodzimierz Maśliński ${ }^{1}$ \\ ${ }^{1}$ Department of Pathophysiology and Immunology, National Institute of Geriatrics, Rheumatology, and Rehabilitation, Warsaw, Poland \\ 2Early Arthritis Clinic, National Institute of Geriatrics, Rheumatology, and Rehabilitation, Warsaw, Poland
}

\begin{abstract}
Objectives: Inflammatory bowel disease (IBD) and spondyloarthritis (SpA) have some overlapping clinical features, i.e. gut and joint inflammation. Cytokines of interleukin 17(IL-17)/IL-23 axis play a pathogenic role in both diseases. Integrins (ITGS) regulate migration of immune cells to inflamed tissues (ITG $\beta 7$ into gut, ITG $\beta 2$ into gut and also to other tissues). In this study, we search for differences in the serum concentrations of these cytokines and integrins between patients suffering from SpA or IBD with and without overlapping symptoms.

Material and methods: Patients with SpA $(n=30), \operatorname{IBD}(n=68)$, and healthy volunteers $(n=28)$ were included in the study. Fourteen SpA patients reported symptoms characteristic for IBD. Spondyloarthritis symptoms were diagnosed in $50 \%$ of IBD patients, while other patients of this group reported arthralgia only. Serum concentrations of IL-17, IL-22, IL-23, ITG $\beta 2$, and ITG $\beta 7$ were measured by specific enzyme-linked immunosorbent assay using commercially available sets. The Mann-Whitney and Spearman's rank tests were used for intergroup comparison and correlation assessment, respectively.

Results: Comparison of patient groups showed significantly higher serum concentrations of IL-17, IL-22, and ITG $\beta 7$ in SpA, and up-regulated levels of IL-23 in IBD patients. Similar differences were observed between patient subgroups, both with and without overlapping symptoms. In SpA but not in IBD patients, serum concentrations of ITG $\beta 7$ inversely correlated $(r=-0.552)$ with C-reactive protein. Conclusions: Patients with SpA and IBD differ in the circulating concentrations of IL-17/IL-23 axis cytokines and ITG $\beta 7$, irrespectively of the presence or absence of overlapping symptoms. Therefore, we conclude that observed differences are attributed rather to underlying than concurrent disease.
\end{abstract}

Key words: cytokines, spondyloarthritis, inflammatory bowel diseases, integrins.

\section{Introduction}

Spondyloarthritis (SpA) and inflammatory bowel disease (IBD) are distinct chronic inflammatory disorders characterised by some degree of overlap between genetic background, clinical symptoms, and consequently the pathogenesis. Clinical chronic IBD, more often Leśniowski-Crohn's disease (L-CD) than ulcerative colitis (UC), develops in 5-10\% of SpA patients, but subclinical gut inflammation, verified by endoscopic and histologic examination, is present in almost half of them, i.e. in $46 \%$ of patients with early SpA and 40-60\% with ankylosing spondylitis [1-3]. On the other hand, arthritis, mostly of SpA subtype, occurs in approximately $15-20 \%$ of L-CD and $10 \%$ of UC patients. In addition, there is a high incidence of asymptomatic sacroiliitis (10-52\%) and in-

Address for correspondence:

Ewa Kontny, Department of Pathophysiology and Immunology, National Institute of Geriatrics, Rheumatology, and Rehabilitation, Spartanska 1, 02-637 Warsaw, Poland, e-mail: ewa.kontny@wp.pl

Submitted: 3.10.2017; Accepted: 28.11.2017 
flammatory low back pain (5-30\%) in IBD patients [4, 5]. Both SpA and IBD are accompanied by various cellular and humoral immune disorders. Among them the interleukin (IL-) 23/IL-17 axis is thought to represent a critical pathway in the pathogenesis of these diseases. Cytokines forming this axis are endowed with important homeostatic functions, but when produced in excess they contribute to and support inflammation-related tissue damage, including gut and joints [6-9].

Numerous cell populations belonging to both innate and adaptive immunity, characterised by expression of IL-23 receptor (IL-23R) and production of IL-17/IL-22, interact at various steps of SpA development. These cells reside both in the gut and in the entheses, contributing to the development of local inflammatory response and its further expansion to distant sites [10, 11]. Infiltration of various immune cells in the inflamed gut, a prominent feature of IBD, is also observed in some AS patients [10]. Leukocyte trafficking to target tissues is governed by adhesion molecules and chemokines. The integrin family comprises leukocyte cell-surface adhesion molecules that play an essential role in migration and extravasation of these cells into tissues. Integrins (ITGS) are heterodimers composed of $\alpha$ and $\beta$ chains that bind to specific ligands at the endothelium. Homing of immune cells to the gut is dependent mostly on $\alpha 4 \beta 7$ integrin that interacts with the mucosal address in-cell adhesion molecule 1 (MAdCAM-1) on endothelial cells, while their retention there is due to binding of $\alpha E \beta 7$ integrin to E-cadherin on epithelial cells [12]. By contrast, b2 subunit (CD18)-comprising integrins are less selective and direct immune cell migration to different target tissues, including gut and joints $[13,14]$. Generation of soluble form of integrins (SITGS) by enzyme-mediated shedding promotes efflux of immune cells from inflammatory sites. In addition, sITGs may act as antagonists limiting local inflammation [15].

Based on this knowledge, we have hypothesised that SpA and IBD patients with overlapping clinical symptoms, i.e. joint and gut inflammation, are more similar than patients devoid of these signs in regard to circulating pool of cytokines and ITGs known to critically contribute to the development of these complications. To verify this presumption the concentrations of IL-17, IL-22, and IL-23 as well as ITGb2 and ITG $\beta 7$ were measured in sera of SpA and IBD patients sub-classified according to the presence or absence of the above overlapping symptoms.

\section{Material and methods}

Two patient cohorts (SpA, $n=30$ and IBD, $n=68$ ) and a group of healthy volunteers $(n=28)$ of similar age were included in the study (Table I). They were recruited from patients admitted to the Early Arthritis Diagnostic Clinic of the National Institute of Geriatrics, Rheumatology, and Rehabilitation (NIGRR) as a part of the routine diagnostic procedures for musculoskeletal complaints or NIGRR staff, respectively. Written, informed consent was obtained from participants before they entered the study. This study was approved by the Ethics Committee of the NIGRR, and all procedures were performed in accordance with the ethical standards and with the 1964 Helsinki Declaration and its later amendments. Clinical evaluation of patients was based on medical history, physical examination, laboratory tests, and recommended radiological examinations.

The diagnosis of SpA was established according to the ASAS (Assessment of SpondyloArthritis International Society) criteria, and disease activity was assessed using BASDAI (Bath Ankylosing Spondylitis Disease Activity Index) [16]. The majority of SpA patients suffered from ankylosing spondylitis (AS) (60\%), while undifferentiated SpA, axial SpA, and psoriatic arthritis (PSA) were less frequent $(17 \%, 13 \%$, and $10 \%$, respectively). Fourteen SpA patients reported symptoms characteristic for IBD, such as recurrent diarrhoea, abdominal pain and cramping, and blood or mucous in stool (subgroup of SpA with IBD symptoms) while others $(n=16)$ had no intestinal symptoms (subgroup of SpA without IBD symptoms). The diagnosis of IBD, UC, and L-CD was established according to criteria described in [17]. From among IBD patients, $57.4 \%$ suffered from UC and $42.6 \%$ from L-CD. Moreover, $50 \%$ of IBD patients had SpA in the form of either AS (27.9\%) or peripheral arthritis (22.1\%) (subgroup of IBD with SpA symptoms) while others were devoid of these complications and reported arthralgia only (subgroup of IBD without SpA symptoms).

Serum was isolated by routine laboratory methods, and serum samples were stored in aliquot at $-70^{\circ} \mathrm{C}$ until assayed. The concentrations of tested factors were measured using commercially available enzyme-linked immunosorbent assay (ELISA) kits specific for: IL-17A/F and IL-23 (both from eBioscience, an Affymetrix Company, San Diego, CA, USA), IL-22 (Abcam, Cambridge, UK), ITGb2, and ITG $\beta 7$ (both from My BioSource, Inc., San Diego, CA, USA). Data were analysed using Statistica 10 software (StatSoft Inc., Tulsa, OK, USA). For intergroup comparison, the Mann-Whitney $U$ test was applied. Correlation was assessed using a Spearman rank test ( $R$ and $p$ values are shown). $P$ values $<0.05$ were considered significant.

\section{Results}

Basic characteristics of the study groups are shown in Table I. In comparison to SpA, the group of IBD pa- 
Table I. Characteristics of the study groups*

\begin{tabular}{|c|c|c|c|c|c|c|}
\hline \multirow[t]{2}{*}{ Parameter } & \multirow{2}{*}{$\begin{array}{l}\text { 1. Healthy } \\
\text { volunteers } \\
(n=28)\end{array}$} & \multirow{2}{*}{$\begin{array}{c}\text { 2. SpA } \\
(n=30)\end{array}$} & \multirow{2}{*}{$\begin{array}{c}\text { 3. IBD } \\
(n=68)\end{array}$} & \multicolumn{3}{|c|}{$P$ value } \\
\hline & & & & 1 vs. 2 & 1 vs. 3 & 2 vs. 3 \\
\hline \multicolumn{7}{|l|}{ Demographics } \\
\hline Age, years & $39(26-56)$ & $42(26-62)$ & $42.5(23-77)$ & 0.49 & 0.22 & 0.59 \\
\hline Gender, female (F) / male (M), $n$ & $22 \mathrm{~F} / 8 \mathrm{M}$ & $13 F / 17 M$ & $41 \mathrm{~F} / 27 \mathrm{M}$ & & & \\
\hline Disease duration, years & & $2(0.5-20)$ & $7(0.2-25)$ & NA & NA & 0.012 \\
\hline Disease activity, (BASDAI), score & & $6.2(3.6-8.1)$ & $6.1(0.6-9.5)$ & NA & NA & 0.41 \\
\hline \multicolumn{7}{|l|}{ Laboratory values } \\
\hline CRP, mg/l & & $21.5(6-84)$ & $10(1-59)$ & NA & NA & $<0.0001$ \\
\hline \multicolumn{7}{|l|}{ Serum concentrations } \\
\hline IL-17 A/F, pg/ml & $20(0-111)$ & $20.6(0-949)$ & $0(0-1234)$ & 0.34 & 0.008 & 0.002 \\
\hline $\mathrm{IL}-22, \mathrm{pg} / \mathrm{ml}$ & $35.2(12-194)$ & $38(0-444)$ & $0(0-111)$ & 0.89 & $<0.0001$ & $<0.0001$ \\
\hline IL-23, pg/ml & $0(0-926)$ & $0(0-2012)$ & $78(0-1082)$ & 0.79 & $<0.0001$ & $<0.0001$ \\
\hline ITGb2, pg/ml & $0(0-100.6)$ & $0(0-283)$ & $0(0-435)$ & 0.65 & 0.92 & 0.66 \\
\hline ITG $\beta 7, \mathrm{ng} / \mathrm{ml}$ & $2.93(1.2-11.4)$ & $3.82(0.37-16.27)$ & $1.5(0-11.8)$ & 0.41 & $<0.0001$ & $<0.0001$ \\
\hline \multicolumn{7}{|l|}{ Medications, \% } \\
\hline NSAIDs & & 100 & & & & \\
\hline Non-biologic DMARDs & & 44.5 & 89.7 & & & \\
\hline Immunosuppressants & & & 38.2 & & & \\
\hline
\end{tabular}

${ }^{\star}$ Except where indicated otherwise, values are the median (min-max values). SpA - spondyloarthritis; IBD - inflammatory bowel disease; BASDAI - the Bath Ankylosing Spondylitis Disease Activity Index; CRP - C-reactive protein; IL - interleukin; ITG - integrin; NSAIDs - non-steroid anti-inflammatory drugs; DMARDs - disease-modifying anti-rheumatic drugs; NA - not applicable.

tients was characterised by longer disease duration but lower values of C-reactive protein (CRP). There was no significant difference between SpA and IBD groups in BASDAI value (Table I). However, $23.5 \%$ of IBD patients had BASDAI score $<3$ while all SpA patients had this score $\geq 3.6$ (data not shown). The majority of SpA patients were positive for HLA-B27 antigen $(84.6 \%$ in subgroup with and $81.8 \%$ in subgroup without IBD symptoms) (data not shown). By contrast, the frequency of HLA-B27 positivity in IBD was lower $(42.4 \%$ in the subgroup with and $11.8 \%$ in the subgroup without SpA complication) (data not shown).

The latter observation is consistent with previously published data [18]. All SpA patients were treated with non-steroidal anti-inflammatory drugs (NSAIDs). The majority of IBD patients and almost half of the SpA patients received non-biologic disease-modifying drugs (DMARDs); none of them were given biologic DMARDs. In addition, almost $40 \%$ of IBD patients were treated with immunosuppressants, and five of them were treated in the past with tumour necrosis factor inhibitors. Sera of patients with IBD contained higher amounts of IL-23, while the concentrations of other cytokines and ITG $\beta 7$ were significantly lower compared to both healthy volunteers and SpA patients. By contrast, there was no significant difference in serum concentrations of tested cytokines and ITGs between the SpA patient group and healthy volunteers. Although serum concentrations of ITGb2 did not differ between tested groups, it should be underlined that detectable levels of this integrin were observed in three healthy volunteers (10.7\%), six IBD patients (8.8\%), and only one SpA patient (3\%) (data not shown). Because of this, ITGb2 was excluded from further analysis.

Comparison of patient subgroups (SpA versus IBD with or without overlapping symptoms) failed to reveal any further differences. As shown in Fig. 1, similarly to the IBD group, also the IBD subgroups were characterised by higher serum concentrations of IL-23 and lower levels of serum IL-17 and IL-22, while the opposite was true for SpA subgroups, regardless of the presence or absence of overlapping symptoms - gut and joint involvement. The only exception was the serum IL-17 A/F level, which was similar in SpA and IBD patients without overlapping signs but was significantly higher in SpA patients with intestinal symptoms than in IBD patients with joint involvement. In addition, the serum ITG $\beta 7$ concentration was significantly higher in SpA than IBD 
A

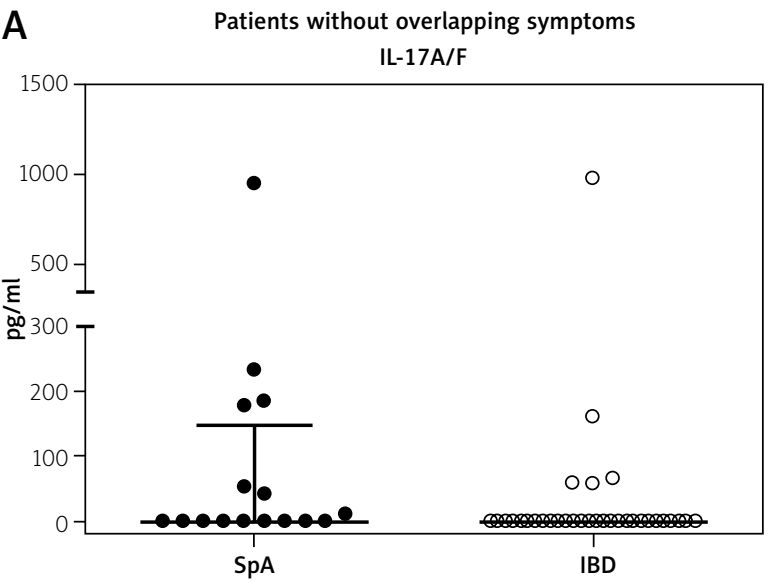

C

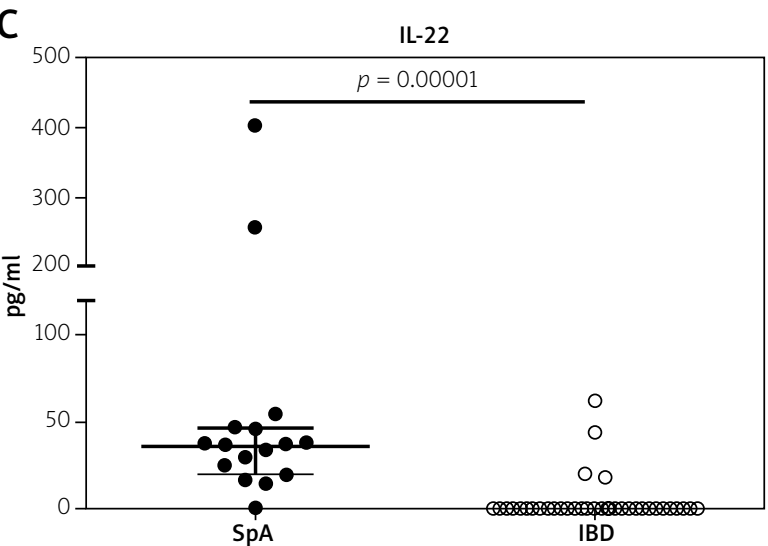

E

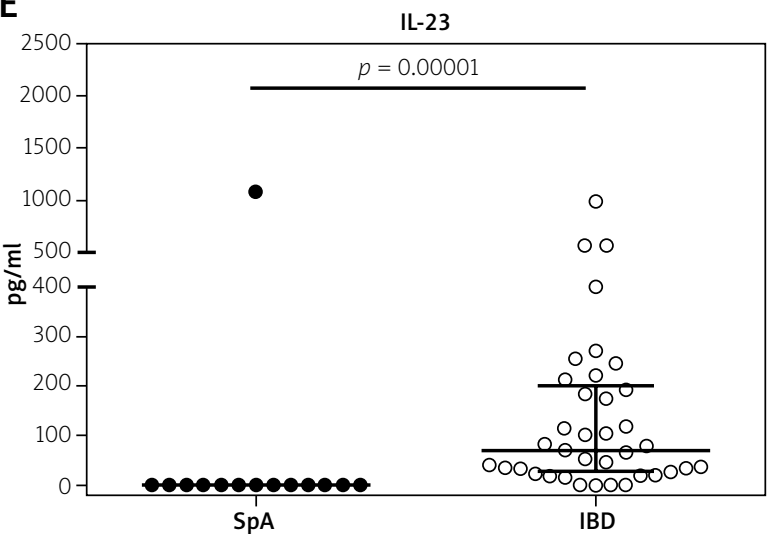

B Patients without overlapping symptoms

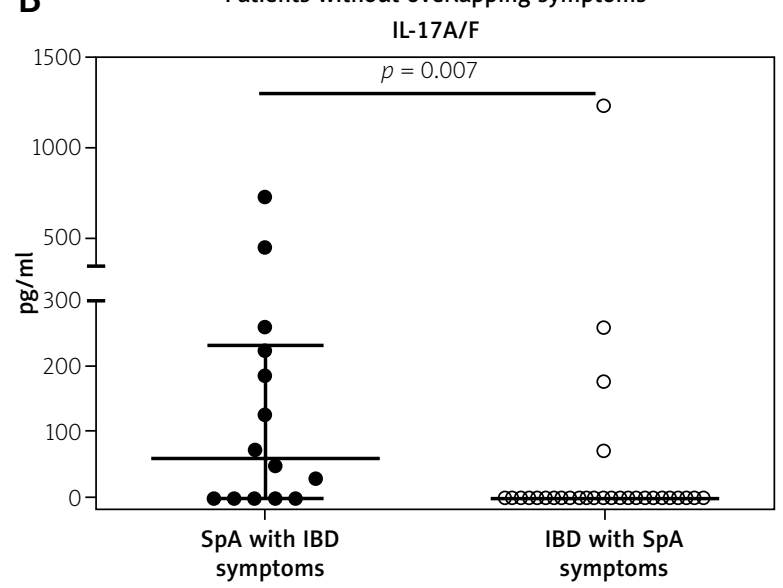

D

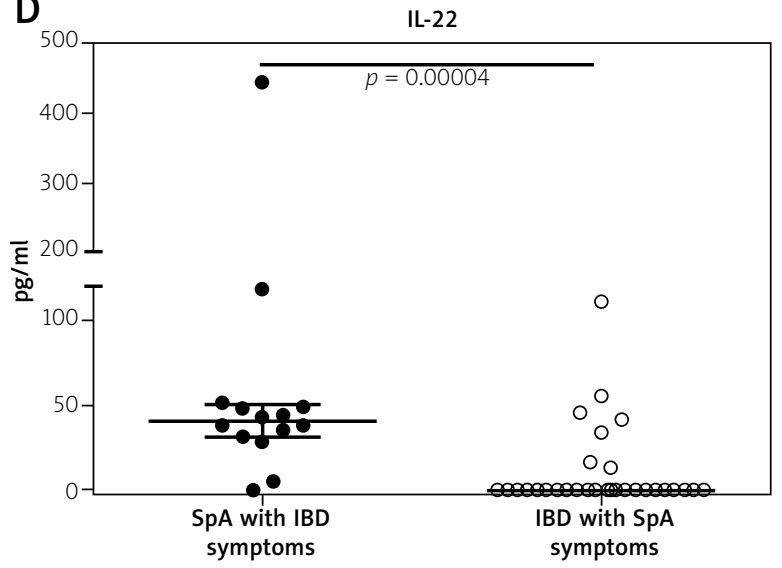

$\mathrm{F}$

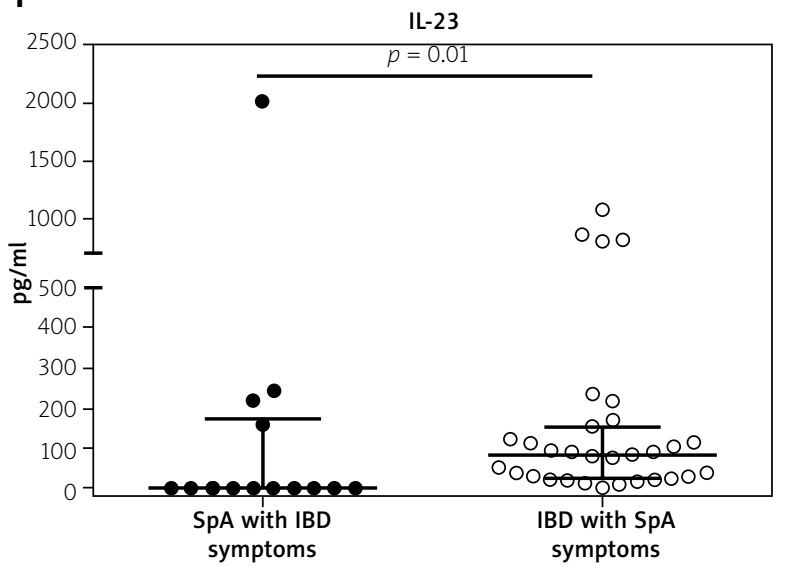

Results are expressed as the median with interquartile range. Each point represents one patient. Left panel - patients without overlapping symptoms; right panel - patients with overlapping symptoms. For statistically significant differences between patients, the groups' $p$ values are shown.

Fig. 1. Serum concentrations of cytokines in spondyloarthritis (SpA) and inflammatory bowel disease (IBD) patient subgroups. 

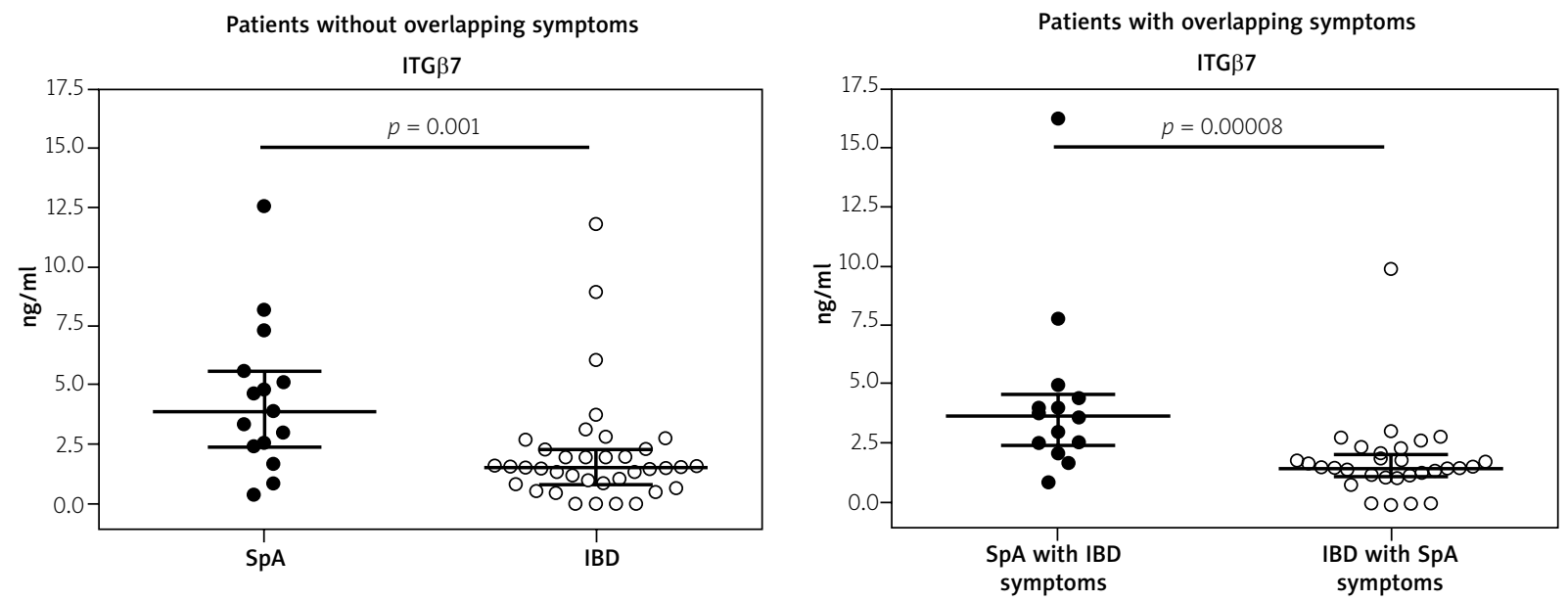

Results are expressed as the median with interquartile range. Each point represents one patient. Left panel - patients without overlapping symptoms; right panel - patients with overlapping symptoms. For statistically significant differences between patients, the groups' $p$ values are shown.

Fig. 2. Serum concentrations of soluble integrin $\beta 7$ (ITG $\beta 7$ ) form in spondyloarthritis (SpA) and inflammatory bowel disease (IBD) patient subgroups.

patients no matter whether the basic disease was or was not complicated with intestine symptoms (Fig. 2) Interestingly, in the SpA group there was significant moderate $(r=-0.522)$ inverse correlation between serum ITG $\beta 7$ and CRP concentrations and a relationship of similar strength was observed in SpA subgroups, especially in patients with IBD symptoms. However, due to the low number of patients in each subgroup these associations did not reach statistical significance. In IBD patients no significant correlation between ITG $\beta 7$ and CRP serum levels was found (Fig. 3).

\section{Discussion}

Based on observations that SpA is a frequent extra-intestinal manifestation in IBD and that subtle gut inflammation is commonly present in SpA patients, the hypothesis of an involvement of common pathogenic mechanisms in these separate clinical entities has been proposed [19]. According to this thesis, IBD-related SpA may originate from the relocation of the immune response primarily induced in the gut-associated lymphoid tissue (GALT), to the joints. Extraintestinal spreading of the immunologic process is proposed to be mediated by immune cells (e.g. T lymphocytes, monocytes/macrophages, innate-like lymphocytes) that express adhesion molecules directing them both to the GALT and joint tissues. On the other hand, accumulating evidence demonstrates that the IL-17/IL-23 cytokine axis is critically involved in many autoimmune diseases, including IBD and SpA [20-24]. Helper T lymphocytes (Th-17, Th-22) and other types of immune cells (e.g. innate-like lymphoid cells - ILCS) produce IL-17 and IL-22 belonging to this cytokine axis. The interleukin 17 family consists of several members, and among them IL-17A and IL-17F share 50\% homology and have proinflammatory potential [20, 24]. In the gut IL-17 and IL-22 play homeostatic functions by supporting the integrity of the epithelial barrier, triggering synthesis of mucous and anti-microbial defensins [20, 21, 23]. These cytokines are also produced by entheseal resident T cells, which play a surveillance role and upon micro-injury launch an immediate inflammatory response and bone regeneration [22, 23]. Interleukin 23, produced by innate immune cells, is the main trigger of IL-17 and IL-22 production and supports also the expansion of committed Th-17 lymphocytes [21, 22].

In the present paper we failed to find significant quantitative differences in the circulating pool of these cytokines between SpA patients and healthy volunteers. However, we noticed higher concentrations of serum IL-23 and lower levels of both IL-17A/F and IL-22 in IBD patients, compared to healthy volunteers and SpA patients (Table I). In SpA cytokines of the IL-17/IL-23 axis are overexpressed mostly in the inflamed tissues (synovial tissue and fluid, entheses, intestine) and quantitative assessment of their circulating pool is inconsistent (higher or similar as in healthy volunteers), indicating their important role in local inflammation and tissue destruction rather than in systemic abnormalities [23, 24]. Consistent with the present results, up-regulated serum concentrations of IL-23 were reported in IBD patients, especially in $\mathrm{L}-\mathrm{CD}[18,25]$. The real contribution of IL-17 and IL-23 to particular disease pathogenesis has 

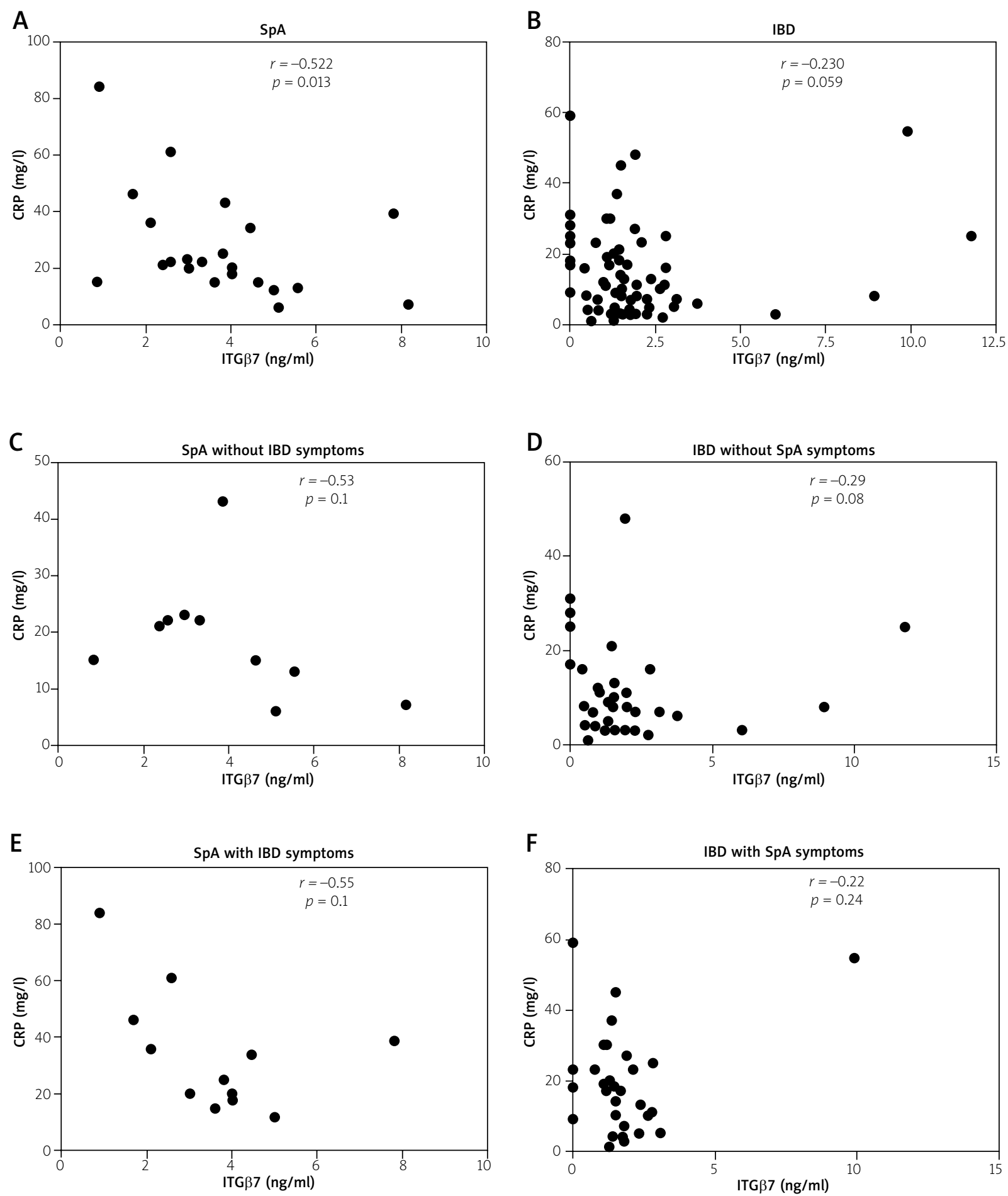

Each point represents one patient. The correlation was assessed in all patients of SpA and IBD groups (upper panels) as well as in patient subgroups without (middle panels) or with (lower panels) overlapping symptoms, using Spearman's Rank test; $R$ and $p$ values are shown.

Fig. 3. Scatter plots showing negative correlation between serum concentrations of integrin $\beta 7$ (ITG $\beta 7$ ) and C-reactive protein (CRP) in spondyloarthritis (SpA) but not in inflammatory bowel disease (IBD) patients. 
been verified by the results of clinical trials with biological drugs specifically neutralising these cytokines. It is clear now that neutralisation of IL-17 is beneficial in SpA but not in IBD patients. By contrast, blocking of IL-23 is effective in L-CD and psoriatic arthritis, but its therapeutic effectiveness in other SpA subtypes needs further investigation [26]. Thus, in general our present results are consistent with these facts.

Interestingly, we found a significant difference in the concentrations of serum ITG $\beta 7$ between IBD and SpA patients on one hand and IBD patients and healthy volunteers on the other (Table I). Integrins containing $\beta 7$ subunit play a central role in the formation of GALT and serve as major molecules homing immune cells to the gut, while b2 chain comprising integrins direct cell migration also to other tissues $[19,27]$. Numerous studies have proven the contribution of ITG $\beta 7$-positive cells to experimentally-induced colitis in laboratory animals, while a role of ITGb2 is less confirmed [27]. Importantly, biological therapies that block ITG $\beta 7$ turned out to be safe and beneficial in IBD and some of them, e.g. monoclonal antibody targeting $\alpha 4 \beta 7$ integrin (vedolizumab VDZ), are approved for the treatment of L-CD [27].

Little is known about the role of ITG $\beta 7$ in SpA. However, a recently published case report describing combination therapy with VDZ and etanercept in a patient suffering from UC with pouchitis and SpA has shown effectiveness of VDZ for pouchitis but not for SpA [28]. The authors of the above report suggest that lymphocytes expressing $\alpha 4 \beta 7$ integrin are probably not associated with the aetiology of SpA. Our present results show measurable concentrations of ITG $\beta 7$ in sera of SpA patients irrespective of gut involvement (Table I, Fig. 2) and an inverse correlation between ITG $\beta 7$ and CRP levels in these patients, not found in the IBD group (Fig. 3). Therefore, it is possible that in SpA the mechanisms that control the availability of ITG $\beta 7$ and protect against excessive migration of immune cells to the intestine are preserved but may become less efficient when systemic inflammation reaches a high level. By contrast, the significant reduction of circulating ITG $\beta 7$ in IBD patients (Table I, Fig. 2) suggests that in this disease, probably due to chronicity of gut inflammation, the above regulatory mechanisms are ineffective. This supposition is supported by previously published data on the relationship between ITG circulating pool and intensity of inflammatory response. First, it is known that part of ITGs may be proteolytically shed from cell membrane into the extracellular environment. Shedding of ITGs may regulate cell migration both directly via decreasing the number of available ITGs with resulting cell detachment and in- directly via adherence to their specific ligands on target tissue $[29,30]$.

Thus, the plasma/serum concentrations of soluble ITGs seem to be a result of a balance between their supply by shedding and depletion by ligand binding. Secondly, in chronic SpA and chronic rheumatoid arthritis (RA), circulating levels of soluble ITGb2 (SCD18) were reported to be decreased and inversely associated with disease activity [29, 30]. In RA patients, normalisation of sCD18 upon successful treatment was also observed [29]. Besides, in animal arthritis models a biphasic course after disease induction was observed with an initial increase followed by a decline [29].

The authors of these reports conclude that decreased plasma levels of sCD18 could reflect transition from early to chronic phase of disease. As for SpA, it is suggested that low levels of plasma sCD18 reflects insufficient CD18 shedding from cells resulting in the failure to block inflammation-induced ligand (ICAM-1) on endothelium and synovium that finally facilitates leukocyte migration to the entheses and joints [30].

In the present study, we did not find a significant difference between tested cohorts in the serum levels of ITGb2 (Table I). The only explanation is a different method used for ITGb2 measurement - we applied ELISA while the cited authors used a time-resolved immunofluorometric assay (TRIFMA) [30]. Despite this, in our study ITGb2 was detectable at measurable levels less frequently in SpA than in other groups (see results), suggesting a similar trend. However, our results on ITG $\beta 7$ circulating pool in SpA and IBD patients resemble the state described above for sCD18 regulation in RA and SpA.

\section{Conclusions}

The present results did not confirm a hypothesis on "immune" similarity of SpA and IBD patients with overlapping symptoms. We report that SpA and IBD patients differ in the circulating concentrations of the IL-17/IL-23 axis cytokines and ITG $\beta 7$. Regardless of the presence or absence of overlapping symptoms (gut and joint inflammation), patients with SpA have higher serum concentrations of IL-17, IL-22, and ITG $\beta 7$, while patients with IBD are characterised by higher serum levels of IL-23. Therefore, we conclude that the above differences are attributed to underlying rather than concurrent disease.

\section{Acknowledgments}

We gratefully acknowledge all patients and healthy volunteers for their contribution to the study. 
This work was financed in parts by the research grant NCN 2012/05/N/NZ5/02839 from the National Science Centre of Poland and the National Institute of Geriatrics, Rheumatology, and Rehabilitation (grant number S/16), Warsaw, Poland.

The authors declare no conflict of interest.

\section{References}

1. Rudwaleit M. Ankylosing spondylitis and bowel disease. Best Pract Clin Rheumatol 2006; 20: 451-471.

2. Van Praet L, Van den Bosch FE, Jacques P, et al. Microscopic gut inflammation in axial spondyloarthritis: a multiparametric predictive model. Ann Rheum Dis 2013; 72: 414-417.

3. Stolwijk C, van Tubergen A, Castillo-Ortiz JD, et al. Prevalence of extra-articular manifestation in patients with ankylosing spondylitis: a systematic review and meta-analysis. Ann Rheum Dis 2015; 74: 65-73.

4. De Vos M. Joint involvement associated with inflammatory bowel disease. Dig Dis 2009; 27: 511-515.

5. Atzeni F, Defendenti C, Ditto MC, et al. Rheumatic manifestations in inflammatory bowel disease. Autoimmun Rev 2014; 13: 20-23.

6. Sarra M, Pallone F, Macdonald TT, et al. IL-23/IL-17 axis in IBD. Inflamm Bowel Dis 2010; 16: 1808-1813.

7. Van Praet L, Van den Bosch F, Mielants $\mathrm{H}$, et al. Mucosal inflammation in spondyloarthritidies: past, present and future. Curr Rheumatol Rep 2011; 13: 409-415.

8. Wendling D, Guillot X, Prati C. The IL-23/Th17 pathway in spondyloarthritis: the royal road? Joint Bone Spine 2015; 82: $1-4$

9. Wendling D. The gut in spondyloarthritis. Joint Bone Spine 2016; 83: 401-405.

10. Ciccia F, Guggino G, Rizzo A, et al. Type 3 innate lymphoid cells producing IL-17 and IL-22 are expanded in the gut, in the peripheral blood, synovial fluid and bone marrow of patients with ankylosing spondylitis. Ann Rheum Dis 2015; 74: 1739-1747.

11. Wendling D, Prati C. Spondyloarthritis: an expanding cast of cellular actors. Joint Bone Spine 2017; doi: 10.1016/j.jbspin.2017.05.001.

12. Zundler S, Becker E, Weidinger C, et al. Anti-adhesive therapies in inflammatory bowel disease - molecular and clinical aspects. Front Immunol 2017; 8: article 891; doi: 10.3389/ fimmun. 2017.00891.

13. Bernstein CN, Sargent M, Rector E. Alteration in expression of b2 integrins on lamina propria lymphocytes in ulcerative colitis and Crohn's disease. Clin Immunol 2002; 104: 67-72.

14. Lowin T, Straub RH. Integrins and their ligands in rheumatoid arthritis. Arthritis Res Ther 2011; 13: 244.

15. Gomez IG, Tang J, Wilson CL, et al. Metalloproteinase-mediated shedding of integrin b2 promotes macrophage efflux from inflammatory sites. J Biol Chem 2012; 287: 4581-4589.
16. Rudwaleit M, van der Heijde D, Landewe E, at al. The Assessment of SpondyloArthritis International Society for classification criteria for peripheral spondyloarthritis and for spondyloarthritis in general. Ann Rheum Dis 2011; 70: 25-31.

17. Gajewski P, Szczeklik A. Choroby jelita grubego. In: Interna Szczeklika - Podręcznik chorób wewnętrznych 2017. Gajewski P (ed.). Medycyna Praktyczna, Kraków 2017.

18. Dmowska-Chalaba J, Kontny E. Inflammatory bowel disease-related arthritis - clinical evaluation and possible role of cytokines. Reumatologia 2015; 53: 236-242

19. Fantini MC, Pallone F, Monteleone G. Common immunologic mechanisms in inflammatory bowel disease and spondyloarthropathies. World J Gastroenterol 2009; 15: 2472-2478.

20. Wallace KL, Zheng LB, Kanazawa Y, et al. Immunopathology of inflammatory bowel disease. World J Gastroenterol 2014; 20: 6-21.

21. Cătăna CS, Neagoe IB, Cozma V, et al. Contribution of the IL-17/IL-23 axis to the pathogenesis of inflammatory bowel disease. World I Gastroenterol 2015, 21: 5823-5830.

22. Lubberts E. The IL-23-IL-17 axis in inflammatory arthritis. Nat Rev Rheumatol 2015; 11: 415-429.

23. Kontny $E$. New aspects of spondyloarthritis pathogenesis. Part III - arthritis, pathological bone remodeling. Reumatologia 2014; 52: 247-254.

24. Miossec P. Update on interleukin-17: a role in the pathogenesis of inflammatory arthritis and implication for clinical practice. RMD Open 2017; 3: e000284. doi: 10.1136/rmdopen-2016-000284.

25. Gheita TA, El Gazzar II, El-Fishawy HS, et al. Involvement of IL-23 in enteropathic arthritis patients with inflammatory bowel disease: preliminary results. Clin Rheumatol 2014; 33: 713-717.

26. Baker KF, Isaacs JD. Novel therapies for immune-mediated inflammatory diseases: What can we learn from their use in rheumatoid arthritis, spondyloarthritis, systemic lupuserythematosus, psoriasis, Chron's disease and ulcerative colitis? Ann Rheum Dis 2017; doi: 10.1136/annrheumdis-2017-211555.

27. Mitroulis I, Alexaki VI, Kourtzelis I, et al. Leukocyte integrins: role in leukocyte recruitment and as therapeutic targets in inflammatory disease. Pharmacol Ther 2015; 147: 123-135.

28. Bethge J, Meffert S, Ellrichmann M, et al. Combinational therapy with vedolizumab and etanercept in a patient with pouchitis and spondyloarthritis. BMJ Open Gastroenterol 2017; 8: e000127.

29. Kragstrup TW, Jalilian B, Keller KK, et al. Changes in soluble CD18 in murine autoimmune arthritis and rheumatoid arthritis reflect disease establishment and treatment response. PLoS One 2016; 11: e0148486.

30. Kragstrup TW, Jalilian B,Hvid M, et al. Decreased plasma levels of soluble CD18 link leukocyte infiltration with disease activity in spondyloarthritis. Arthritis Res Ther 2014; 16: R42. 